\title{
ON SINGULAR HAMILTONIANS: THE EXISTENCE OF QUASI-PERIODIC SOLUTIONS AND NONLINEAR STABILITY
}

CHJAN C. LIM

In this note, we announce results concerning the existence of quasiperiodic solutions for a class of singular Hamiltonians, and the nonlinear stability of singularities (as opposed to equilibria). A third result concerns the existence of bounded, connected and open invariant sets in the neighborhood of singularities. Historically, the existence of quasi-periodic solutions and nonlinear stability of equilibria for Hamiltonian systems are established via the KAM theorems [A1, A2, M1, M2]. When the question of quasi-periodic behavior in the neighborhood of singularities is the issue (as in the restricted 3-body problem near one of the primaries), the singularity is transformed into an equilibrium by an appropriate regularization (cf. the classic papers [C and M3]). To the best of our knowledge, there is no regularization procedure for the class of singular Hamiltonians discussed here. We therefore adopt a different approach.

Existence of quasi-periodic solutions. We consider the Hamiltonian,

$$
H\left(q_{1}, \ldots, q_{n} ; p_{1}, \ldots, p_{n}\right)=\sum_{j=1}^{m} K_{j} \log \left|f_{j}\left(z_{1}, \ldots, z_{n}\right)\right|
$$

with the following properties

(A) $\quad z_{j}=q_{j}+i p_{j}$ and $K_{j}$ are real coefficients,

(B) $\quad f_{j}\left(z_{1}, \ldots, z_{n}\right)$ are entire functions of $n$ complex variables,

(C) the zero level sets, $M_{j}=f_{j}^{-1}(0)$ are complex hyperplanes which intersect at a unique point, $\mathbf{z}^{*}$ (without any loss in generality, $\mathbf{z}^{*}=\mathbf{0}$ ).

This class of Hamiltonians arise in several applications notably $N$-body problems. In these problems $m=N(N-1) / 2$, the number of edges in the complete graph consisting of $N$ vertices; $n$ equals $N-1$, the number of independent degrees of freedom after the usual reduction in the presence of translational symmetry.

Received by the editors February 8, 1988.

1980 Mathematics Subject Classification (1985 Revision). Primary 34C28, 34D99, 70H05; Secondary $76 \mathrm{C} 05,70 \mathrm{~F} 10$. 
It is convenient to use the complex formulation given below; the branch of the logarithm function is immaterial here

$$
H_{c}\left(z_{1}, \ldots, z_{n}\right)=\sum_{j=1}^{m} K_{j} \log \left[f_{j}\left(z_{1}, \ldots, z_{n}\right)\right] .
$$

The complex equations of motion are

$$
\dot{\bar{z}}_{j}=i \frac{\partial H_{c}}{\partial z_{j}} \quad j=1, \ldots, n .
$$

Taking real and imaginary parts, one recovers the usual Hamilton's equations

$$
\dot{q}_{j}=\frac{\partial H}{\partial p_{j}}, \quad \dot{p}_{j}=\frac{\partial H}{\partial q_{j}} \quad j=1, \ldots, n .
$$

In order to apply the KAM theorem in a neighborhood of the branch point $\mathbf{z}^{*}$, we first construct special open sets in phase space called cone sets that are bounded away from the singular manifolds defined in (C) and satisfy an additional condition on the variables $z_{j}$,

$$
\begin{aligned}
M_{\varepsilon}^{\delta}= & \left\{\mathrm{z}=\left(z_{1}, \ldots, z_{n}\right)|0<| z_{1}|<\delta| z_{2}\left|<\delta^{2}\right| z_{3} \mid<\cdots\right. \\
& \left.<\delta^{n-1}\left|z_{n}\right|<\delta^{n} \varepsilon\right\}, \quad \delta=O(\varepsilon) .
\end{aligned}
$$

Now Lemma 1 states that the Hamiltonian (1) can be written as a small perturbation of an integrable term in the cone sets.

LEMMA 1. Consider the Hamiltonian (1) with the additional assumptions,

$$
\begin{aligned}
& \text { for all } k \leq m,\left(\partial f_{k} / \partial z_{h}\right)\left(\mathbf{z}^{*}\right) \neq 0 \text { for some } h \leq k \text {, but } \\
& \left(\partial f_{k} / \partial z_{h}\right)\left(\mathbf{z}^{*}\right)=0 \text { for } h>k .
\end{aligned}
$$

Then, in the open sets (5), the Hamiltonian (1) is

(i) a $O(\varepsilon)$ perturbation of the decoupled Hamiltonian,

$$
H_{0}(\mathbf{z})=\sum_{j=1}^{n} \Gamma_{j} \log \left|z_{j}\right|
$$

(ii) the perturbation is real analytic in the action variables $\left(J_{1}, \ldots, J_{n}\right)$ and $2 \pi$-periodic in the angles $\left(\theta_{1}, \ldots, \theta_{n}\right)$ where $z_{h}=\sqrt{J_{h}} e^{i \theta_{h}}$.

Sketch of Proof. In the sets $M_{\varepsilon}^{\delta}$ which lie in an $\varepsilon$-ball centered at the origin $\mathbf{z}^{*}=\mathbf{0}$ of $\mathbf{R}^{2 n}$, Taylor expansions for the entire functions $f_{j}(\mathbf{z})$ give (after using the complex formulation),

$$
H=\sum_{j=1}^{m} K_{j} \log \left|z_{h(j)}\right|+\sum_{j=1}^{m} K_{j} \log \mid(1+O(\varepsilon) \mid .
$$

The function $h(j)<j$ is defined to be the largest index of the complex variables $z_{i}$ for which $\left(\partial f_{j}(\mathbf{0}) / \partial z_{i}\right) \neq 0$. Condition (6) implies $h(j)$ is well 
defined for $1 \leq j \leq m$. Thus the completely integrable term, $H_{0}$, is given by (7) where $\bar{\Gamma}_{j}=\sum_{i \in \chi_{j}} K_{i}$ and the set $\chi_{j}=\{i \in\{j, \ldots, m\} \mid h(i)=j\}$. From (8), it is clear that the perturbation is $O(\varepsilon)$ in $M_{\varepsilon}^{\delta}$ and conclusion (ii) follows immediately.

Next an application of the KAM theorem [A1, M1] gives a condition on the coefficients $\Gamma_{j}$ which guarantees the existence of quasi-periodic solutions for the original Hamiltonian (1).

THEOREM 1. If the coefficients $\Gamma_{j}$ in $H_{0}(7)$ satisfy the condition

$$
\prod_{j=1}^{n} \Gamma_{j} \neq 0
$$

then there exists a positive measure set of quasi-periodic solutions for (1) in a sufficiently small neighborhood of the point $\mathbf{z}^{*}(=\mathbf{0})$.

SKeTCH OF PROOF. For sufficiently small $\varepsilon$, Lemma 1 implies that all except one of the KAM hypotheses are satisfied in $M_{\varepsilon}^{\delta}$. It remains to verify that the nondegeneracy condition [A1, M1] holds when condition (9) is valid (which is an easy computation).

Nonlinear stability of singularities. For two degrees of freedom, the stability of Hamiltonian singularities can be discussed in a manner that parallels the usual nonlinear stability theory of equilibria [A2, M1]. First we need a definition.

Definition. A singularity, $\mathbf{z}^{*}=\left(z_{1}^{*}, z_{2}^{*}\right)$ of a given Hamiltonian $H=$ $H\left(z_{1}, z_{2}\right)$ is nonlinearly stable if given $\varepsilon>0, \exists \delta>0$ such that if $\left|\mathbf{z}(0)-\mathbf{z}^{*}\right|<$ $\delta$ then $\left|\mathbf{z}(t)-\mathbf{z}^{*}\right|<\varepsilon \forall t \in(-\infty, \infty)$. The norm $|\mathbf{z}|$ is given by

$$
\left(\left|z_{1}\right|^{2}+\left|z_{2}\right|^{2}\right)^{1 / 2}
$$

where $\left|z_{j}\right|$ is the modulus of the complex variable $z_{j}$ for $j=1,2$. We consider two types of singularities here, namely the logarithmic branch point and the simple pole. Results for other singularities are discussed in [L1, L2].

TheOREM 2. Consider the Hamiltonian

$$
H=\omega_{1} \log \left(J_{1}\right) \pm \omega_{2} \log \left(J_{2}\right)+H_{1}\left(J_{1}, J_{2}, \theta_{1} \theta_{2}\right)
$$

in action-angle form where $\omega_{1}, \omega_{2}$ are positive real numbers. $H_{1}$ is real analytic in $J_{1}, J_{2}, 2 \pi$-periodic in $\theta_{1}, \theta_{2}$ and $O\left(J_{1}, J_{2}\right)$ in the neighborhood of the singularity $\left(J_{1}, J_{2}\right)=(0,0) \equiv \mathbf{0}$.

(i) In the plus case, for all $\omega_{1}, \omega_{2} \mathbf{0}$ is nonlinearly stable.

(ii) In the minus case, 0 is nonlinearly stable if $\omega_{1} \neq \omega_{2}$.

REMARKS. The singularity at the origin $\mathbf{0}$ is the unique intersection point of the singular manifolds defined by $J_{1}=0$ and $J_{2}=0$ respectively.

SKETCH OF PROOF FOR THE PLUS CASE. The isoenergetic version of the KAM theorem is applied to the product of punctured disks for the complex variables $z_{1}$ and $z_{2}$ (where $z_{h}=\sqrt{J_{h}} e^{i \theta_{h}}$ ), i.e.

$$
\left(D_{1}^{0} \times D_{2}^{0}\right)(\varepsilon)=\left\{\left(z_{1}, z_{2}\right) \in \mathbf{C}^{2} \mid 0<J_{1}, 0<J_{2}, J_{1}+J_{2}<\varepsilon^{2}\right\} .
$$


On $M_{h}$, the 3-dimensional isoenergetic manifold for

$$
H_{0}\left(J_{1}, J_{2}\right)=\log \left(J_{1}^{\omega_{1}} J_{2}^{\omega_{2}}\right),
$$

we have $J_{1}^{\omega_{1}} J_{2}^{\omega_{2}}=e^{-h}$. For $h$ sufficiently large, $M_{h}$ intersects $D_{1}^{0} \times D_{2}^{0}$ with $\varepsilon \ll 1 . M_{h}$ is foliated by tori that are parametrized by $J_{1}\left(J_{2}\right.$ can be solved from the above relation) but note that as $J_{1} \searrow 0, J_{2} \nearrow \infty$. Thus the entire interior of these tori is not contained in the $\varepsilon$-neighborhood, (10). This motivates the key idea of trapping an orbit in the annular region between two KAM tori on the same energy surface. Provided the isoenergetic form of the nondegeneracy condition holds the KAM theorem [A1, M1] implies that a large set of invariant tori for the full Hamiltonian, $H$ can be found in sufficiently small $\varepsilon$-neighborhood of the origin. Now given by $\varepsilon>0, \exists$ a sufficiently small $\delta>0$ such that a point in the $\delta$ neighborhood (in the sense of (10)) is contained in the annular region between $2 \mathrm{KAM}$ tori on the same $M_{h}$ for some energy $h$. This annular region is an invariant subset of the $\varepsilon$-neighborhood thus establishing the stability of 0 . It remains to verify that the isoenergetic nondegeneracy condition holds for $H_{0}$ i.e. the determinant of the bordered Hessian is nonzero which is an easy computation.

The proofs for part (ii) above and Theorem 3 are similar.

THEOREM 3. Consider the Hamiltonian

$$
H=\frac{\omega_{1}}{J_{1}}+\frac{\omega_{2}}{J_{2}}+H_{1}\left(J_{1}, J_{2}, \theta_{1}, \theta_{2}\right)
$$

where $H_{1}$ satisfies the same conditions as in Theorem 2. The singularity $\mathbf{0} \equiv(0,0)$ is nonlinearly stable for all values of $\omega_{1}, \omega_{2}$.

These results should be contrasted to the stability results for elliptic equilibria of Hamiltonian systems [A1, M1, MS] where typically, additional terms in the normal form are required in order to satisfy the nondegeneracy conditions.

Bounded, connected and open invariant sets. Consider a 2 degrees of freedom Hamiltonian which has the form of a small perturbation

$$
H\left(J_{1}, J_{2}, \theta_{1}, \theta_{2}\right)=H_{0}\left(J_{1}, J_{2}\right)+H_{1}\left(J_{1}, J_{2}, \theta_{1}, \theta_{2}\right)
$$

only in a restricted neighborhood of the origin in $\mathbf{R}^{4}$. This is the case for the Hamiltonian (1) discussed above. The existence of quasi-periodic solutions is thus guaranteed in the cone sets (5) but not for a full neighborhood of the singularity, $\mathbf{z}^{*}(=0)$ by Theorem 1 . Hence, the nonlinear stability theory is not applicable. On the other hand, Theorem 4 gives the construction of invariant sets that are open in $\mathbf{R}^{4}$ and lie in the neighborhood of the origin. In Theorem 4 , the origin can be an elliptic equilibrium point or a singularity such as a logarithmic branch point or a pole. This theorem implies that quasi-periodic solutions in the neighborhood of the origin become bounded orbits that remain near the origin when perturbed slightly. 
THEOREM 4. Consider the Hamiltonian (11) such that $H_{1}$ is real-analytic in $J_{1}, J_{2}, 2 \pi$-periodic in $\theta_{1}, \theta_{2}$ and $O(\varepsilon)$ only in some open subset of an $\varepsilon$-neighborhood of the origin. If $H_{0}\left(J_{1}, J_{2}\right)$ satisfies the isoenergetic nondegeneracy KAM condition, then there exists bounded, open and connected invariant sets in a sufficiently small neighborhood of the origin.

SKETCH OF PROOF. On an isoenergetic manifold $M_{h}$ which intersects the open subset in which $H_{1}$ is $O(\varepsilon)$, choose an invariant torus $T(1)$; clearly the bordered Hessian at $T(1)$ is bounded away from zero. $T(1)$ has rotation number $f^{*}=\omega_{1}^{*} / \omega_{2}^{*}$ which is strongly nonresonant. By continuity of the bordered Hessian, the sufficiently short segment of frequencies

$$
\left(\omega_{1}, \omega_{2}\right)=\left(t \omega_{1}^{*}, t \omega_{2}^{*}\right), \quad t \in(1-\delta, 1+\delta)
$$

with constant rotation number, $f^{*}$ has a corresponding curve of invariant tori, $\{T(t)\}$ which passes through $T(1)$ on $M_{h}$. The union of the interior

$$
T=\bigcup_{t} \operatorname{int}(T(t))
$$

is a connected and bounded invariant set for the Hamiltonian system. It remains to show that the curve of the tori $\{T(t)\}$ does not lie in one 3-dimensional energy manifold. This follows from the isoenergetic nondegeneracy condition.

Applications. We end this note by a brief discussion of the applications. Theorems 1 and 2 can be applied to $N$-body problems with logarithmic potential interaction such as vortex dynamics $[\mathbf{L 1}, \mathbf{L 2}, \mathbf{L 3}, \mathbf{L 4}]$ and twodimensional charge dynamics (the guiding center models) [MO]. Theorem 4 is useful for proving the observability (or persistence) of clusters in $\mathrm{N}$ body problems.

\section{REFERENCES}

[A1] V. I. Arnold, Math. methods of classical mechanics, Springer-Verlag, Berlin and New York, 1980.

[A2] _ _ Russian Math. Surveys 18(5) (1963), 9.

[C] C. Conley, Comm. Pure Appl. Math. 16 (1963), 449-467.

[L1] Chjan C. Lim, Singular manifolds and quasi-periodic solutions of Hamiltonians for vortex lattices, Physica D 30 (1988), 343.

[L2] _ _ Symplectic techniques for vortex N-body problems, Comm. Math. Phys. (submitted).

[L3] _ _ Quasi-periodic dynamics of desingularized vortex models, Physica D (1988).

[L4] _ On Hamiltonian singularities and applications, Proc. of the Int'l Conf. on Theory and Applications of Diff. Eqns., March 21-25, 1988, Columbus, Ohio.

[M1] J. Moser, Stable and random motions in dynamical systems, Princeton Univ. Press, Princeton, N.J., 1973.

[M2] —, Nachr. Akad. Wiss. Gott. Math. Phys. Kl. 2 (1982), 1.

[M3] _ Comm. Pure Appl. Math. 23 (1970), 609-636.

[MO] G. Joyce and D. Montgomery, J. Plasma Phys. 10 (1973), 107.

[MS] K. Meyer and D. Schmidt, J. Differential Equations 62 (1986), 222-336. 
[MW] J. Marsden and A. Weinstein, Physica D 7 (1983), 305.

[Z] S. L. Ziglin, Dokl. Akad. Nauk SSSR 250 (1980), 1296-1300; Soviet Math. Dokl. 21 (1980), 296-299.

Department of Mathematics, University of Michigan, Ann Arbor, Michigan 48109

Current address: Institute for Mathematics and its Applications, University of Minnesota, Minneapolis, Minnesota 55455 